\title{
Ortner's Syndrome: Secondary Laryngeal Paralysis Caused by a Great Thoracic Aorta Aneurysm
}

\author{
Ana Claudia Alves Zangirolami ${ }^{1} \quad$ Frederico Vieira de Oliveira $^{1} \quad$ Miguel Soares Tepedino ${ }^{1}$ \\ ${ }^{1}$ Department of Otorhinolaryngology, Policlinica de Botafogo, Rio de \\ Janeiro, Brazil \\ Address for correspondence Ana Claudia Alves Zangirolami, MD, \\ Department of Otorrinolaringologia, Policlinica de Botafogo, Av \\ Pasteur 72, 1 andar, Rio de Janeiro 22290240, Brazil \\ Int Arch Otorhinolaryngol 2015;19:180-182. \\ (e-mail: anacrudy@yahoo.com.br).
}

\begin{abstract}
Introduction Recurrent laryngeal nerve injury caused by cardiovascular disease is a rare condition, and often it is the only prominent sign of an imminent break of an aortic artery aneurysm.

Objective To report left laryngeal paralysis caused by a great aortic arch aneurysm and to highlight the importance of an otorhinolaryngologic evaluation along with a thoracic radiologic study.

Resumed Report A 42-year-old man complained of thickness of his voice and dysphagia for 3 months, but no thoracic pain or other relevant complaints. Video laryngoscopy revealed immobility of his left vocal fold in the paramedian position. Imaging was obtained for investigation, including magnetic resonance imaging of his thorax, which showed a fusiform aneurysm in the aortic arch, leading to recurrent compression of the left laryngeal nerve. The patient was successfully treated with endovascular repair of the aneurysm. At 2-month follow-up, there was still no recovery of the laryngeal mobility.

Keywords

- vocal cord palsy

- arch of the aorta

- dysphonia

Conclusion An aortic artery aneurysm can suddenly break, requiring emergency heart surgery, and the results can be fatal in many cases. We suggest routine exam of the vocal folds in all patients with a heart condition, and we review the literature and suggest the use of imaging to reduce the number of emergency procedures.
\end{abstract}

\section{Introduction}

Ortner's syndrome, or cardiovocal syndrome, was introduced by Norbert Ortner in 1897, who described three cases of left vocal fold immobility due to the recurrent compression of the laryngeal nerve, caused by dilatation of the left atrium in patients with mitral valve stenosis. ${ }^{1}$ Since then, the term Ortner's syndrome has been used to describe any nonmalignant intrathoracic heart condition that results in recurrent compression of the laryngeal nerve. The possible causes are left ventricular failure, atrial septum failure, arterial canal persistency, primary pulmonary hypertension, pulmonary artery relapsing embolism, left ventricular aneurysm, and other types of aortic aneurysm that cause vocal fold paralysis. Thoracic aortic artery aneurysm represents $5 \%$ of the cases. ${ }^{2,3}$ The prevalence of the cardiac alterations that characterize Ortner's syndrome is not discussed in the literature, because its manifestation is linked to many causes and depends on the direct compression of the nerve. The left recurrent laryngeal nerve is the most frequently affected, due to its longer course bypassing the aortic arch, compared with the right one, which goes around the subclavian artery. received

June 3, 2014 accepted

November 3, 2014

published online

January 5, 2015
DOI http://dx.doi.org/

10.1055/s-0034-1397331. ISSN $1809-9777$.
Copyright $\odot 2015$ by Thieme Publicações License terms Ltda, Rio de Janeiro, Brazil

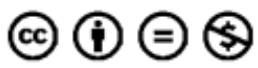




\section{Literature Review}

The vocal fold immobility in Ortner's syndrome can be associated with involvement of the vagal nerve or the recurrent laryngeal nerve, from the jugular foramen to the entrance of the larynx. It has many etiologies according to the lesion's location, including trauma during thoracic (53.1\%) and heart surgeries (28.5\%), extended intubation (16.7\%), neoplasms (32\%), central nervous system dysfunction (8\%), and metabolic and infectious (3\%), idiopathic (16\%), and traumatic (11\%) causes. ${ }^{2}$ Aortic aneurysm is an uncommon cause for vocal cord paralysis, described in only $5 \%$ of the patients with this vascular condition, and the involvement of the ascendant aorta and the aortic arch is also infrequent. ${ }^{2-4}$

\section{Case Report}

A 42-year-old man born in Rio de Janeiro presented to the Otorhinolaryngologic Service complaining of dry cough and thickness of his voice of 3 months' duration, combined with progressive dysphagia from solid to liquid. Otorhinolaryngologic physical exam discovered no alterations. Indirect laryngoscopy revealed left vocal fold paralysis at the paramedian position (-Fig. 1). Thoracic X-ray was requested, which showed a bulge adjacent to the aortic area; neck and cranium computerized tomography showed no alterations. Magnetic angioresonance of the mediastinum revealed a fusiform aneurysm of the thoracic aorta with laminar mural thrombi, measuring $7 \mathrm{~cm}$ in diameter and extending $\sim 9.5 \mathrm{~cm}$, with no evidence of dissection. The heart had normal dimensions (-Figs. 2, 3, and 4). These results indicated left recurrent laryngeal nerve paralysis by compression of the aortic artery. The patient was referred to the cardiovascular surgeon for surgical treatment of the aortic aneurysm, which was accomplished by means of endovascular surgery. Two months postoperatively, the patient still had dysphonia and vocal fold immobility.



Fig. 1 Laryngoscopy image showing paramedian left vocal fold paralysis.

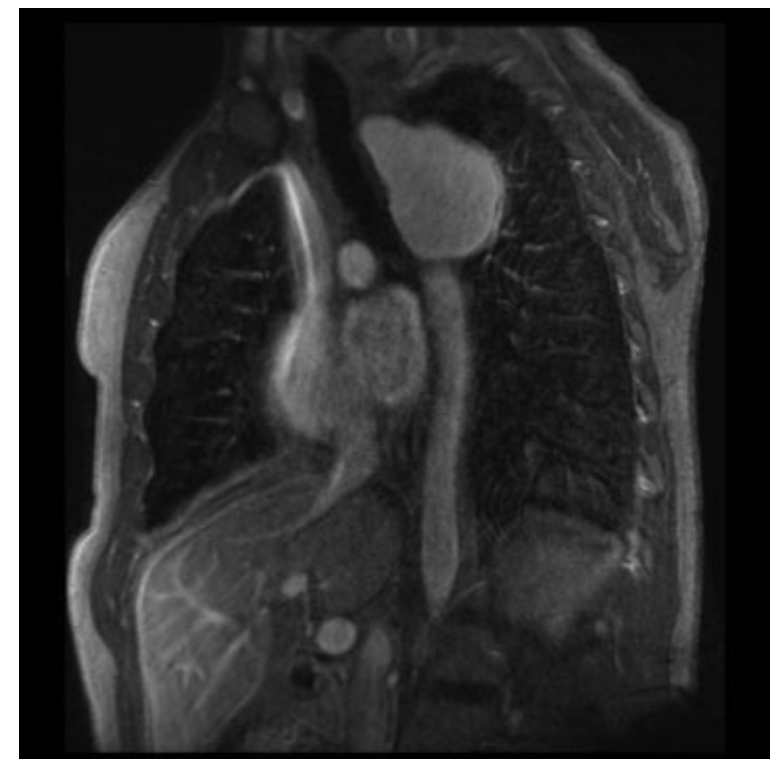

Fig. 2 Magnetic resonance T1-weighted image, coronal section, showing aneurysmal dilatation of the aorta arch.

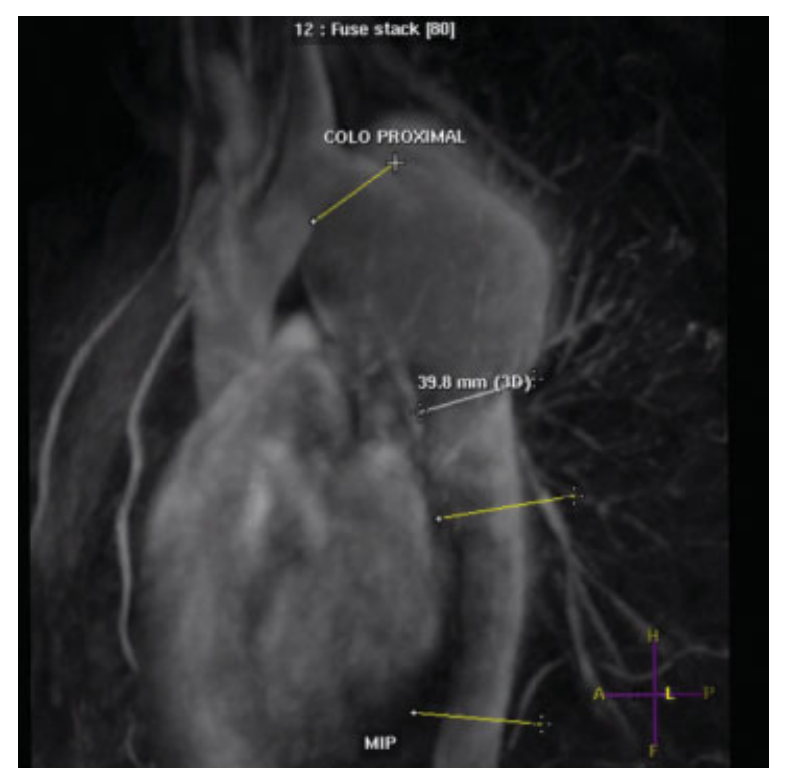

Fig. 3 MRI examination T1-weighted image, examination in oblique profile for rotational rebuild with paramagnetic contrast, showing a fusiform aneurysm extending from the post emerging segment of the subclavian artery and demonstrating mural thrombi and misuse of adjacent structures. Note also topography of vagus nerve. Abbreviation: $A$, anterior; F, feet; $\mathrm{H}$, head; L, left; MIP, maximum intensity projection; P, posterior.

\section{Discussion}

The left recurrent laryngeal nerve, due to its intimate anatomic relation to the aortic arch, left lung apex, trachea, esophagus, left pulmonary artery, and mediastinal lymph nodes, is particularly vulnerable to lesions. The most frequent causes of paralysis are tumors of the lung, esophagus, and thyroid. It has also been reported as a possible complication from other cardiovascular diseases, including pulmonary 


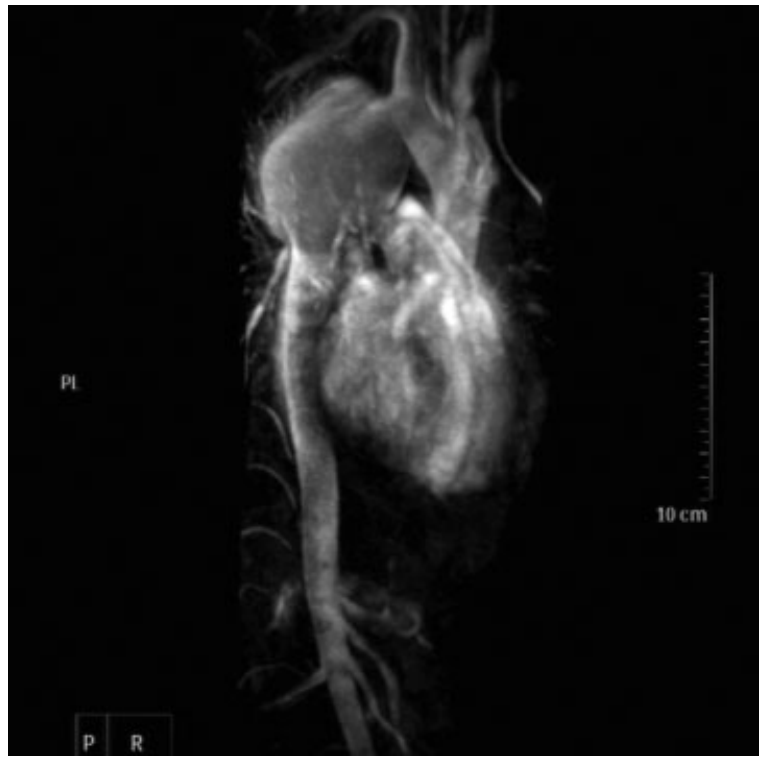

Fig. 4 MRI examination T1-weighted image, coronal cut for rotational rebuild with paramagnetic contrast, showing a fusiform aneurysm extending from the post emerging segment of the subclavian artery and demonstrating mural thrombi and misuse of adjacent structures. Note also topography of vagus nerve. Abbreviation: PL, posterior left; $\mathrm{PR}$, posterior right.

hypertension, interatrial communication, and patency of the arterial canal. 5,6 The mechanism of left recurrent laryngeal nerve paralysis remains unclear. However, it is assigned to the compression of this nerve, which can get hooked around the ligament arteriosus between the pulmonary artery and the aortic aneurysm. Recurrent laryngeal nerve paralysis in patients with cardiovascular diseases demands a thorough examination, because it suggests possible dilatation of the aneurysm. ${ }^{7}$ For diagnosis, in addition to the anamnesis and the physical examination, imaging must be used to delimitate the location and diameter of the aneurysm, with thoracic computed tomography and magnetic resonance able to sense lesions nearly $100 \%$ of the time. It is also vital that in patients with laryngeal complaints (vocal, breathing, or swallowing disturbances), a videolaryngoscopy is used first to identify the vocal fold position, if there is an intrinsic lesion, imaging is used to investigate the entire neural path of the vagal nerve, from its emergence on the cranium base to the recurrent laryngeal nerve emission in the thorax. ${ }^{8}$ Recurrent laryngeal nerve paralysis prognosis depends on the level and time elapsed from the compression of the nerve. Stoob et al reported the first case of curing the laryngeal paralysis of a patient 1 year after surgically correcting the aneurysm by endovascular means. ${ }^{4}$ However, in most of the cases reported in literature, there was no resolution of the thickness of the voice. Deaths by surgical complications or breaking of the aneurysm were also reported. ${ }^{2,3,9}$ Patients with laryngeal paralysis in the paramedian position must be submitted to speech therapy to use the contralateral musculature to compensate for the glottal failure. Surgical treatment can be indicated for patients with important refractory vocal symptoms on speech therapy or for those with imminent risk of bronchoaspiration. $^{10,11}$

\section{Final Comments}

An aortic artery aneurysm can suddenly break, indicating emergency heart surgery, which often has fatal results. We suggest routine exam of the vocal folds in all patients with a heart condition.

\section{References}

1 Chan P, Lee CP, Ko JS, et al. Cardiovocal (Ortner's) syndrome: left recurrent laryngeal nerve palsy associated with cardiovascular diseases. Eur J Med 2004;11:69-70

2 Yuan SM. Hoarseness subsequent to cardiovascular surgery, intervention, maneuver and endotracheal intubation: the so-called iatrogenic Ortner's (cardiovocal) syndrome. Cardiol J 2012; 19(6):560-566

3 Ohki M. Thoracic saccular aortic aneurysm presenting with recurrent laryngeal nerve palsy prior to aneurysm rupture: a prodrome of thoracic aneurysm rupture? Case Rep Otolaryngol 2012; 2012:367873

4 Camishiou RC, Gibbon JH, Pierucci L, et al. Paralysis of the left recurrent laryngeal nerve secondary to mitral value disease: report of two cases and literature review. Ann Surg 1966; 163(6):818-827

5 Fennessy BG, Sheahan P, McShane D. Cardiovascular hoarseness: an unusual presentation to otolaryngologists. J Laryngol Otol 2008;122(3):327-328

6 Stoob K, Alkadhi H, Lachat M, Wildermuth S, Pfammatter T. Resolution of hoarseness after endovascular repair of thoracic aortic aneurysm: a case of Ortner's syndrome. Ann Otol Rhinol Laryngol 2004;113(1):43-45

7 Gupta P, Sharma S. Ortner's syndrome secondary to aortic aneurysm. Ann Acad Med Singapore 2012;41(1):40-41

8 Gulel O, Elmali M, Demir S, Tascanov B. Ortner's syndrome associated with aortic arch aneurysm. Clin Res Cardiol 2007; 96(1):49-50

9 Subramaniam V, Herle A, Mohammed N, Thahir M. Ortner's syndrome: case series and literature review. Braz J Otorhinolaryngol 2011;77(5):559-562

10 Mathai J, Swapna UP. Hoarseness -as a presenting feature of aortic arch aneurysm. Indian J Otolaryngol Head Neck Surg 2006;58(3): 309-310

11 Veldtman GR, Dearani JA, Warnes CA. Low pressure giant pulmonary artery aneurysms in the adult: natural history and management strategies. Heart 2003;89(9):1067-1070 